\title{
De la subordinación asalariada a la caridad neoliberal: análisis crítico de la ley de inclusión laboral de personas con discapacidad en Chile
}

\section{From wage subordination to neoliberal charity: critical analysis of the labor inclusion law for people with disabilities in Chile}

Juan Carlos Cea Madrid

Universidad de Chile, Chile

\begin{abstract}
RESUMEN El presente artículo analiza la entrada en vigencia de la Ley $\mathrm{N}^{\circ}$ 21.015 "Incentiva la inclusión de personas con discapacidad al mundo laboral". Esta normativa tiene por finalidad mejorar la empleabilidad de las personas con discapacidad en entornos laborales abiertos por medio de la reserva del $1 \%$ de la cuota de empleos en empresas con 100 o más trabajadores. Al respecto, se examinan las limitaciones de esta política de acceso al empleo para la promoción del derecho al trabajo y la configuración de sociedades más inclusivas. De acuerdo con un análisis crítico de la implementación de la iniciativa legal en el sector privado, se observan brechas salariales, etarias y de género en la incorporación y permanencia de las personas con discapacidad en el mercado de trabajo. Finalmente, se concluye que las estrategias de inserción laboral en el campo de la discapacidad no cuestionan las estructuras de poder en la sociedad capitalista y se integran a las bases del modelo neoliberal en el Chile contemporáneo.
\end{abstract}

PALABRAS CLAVES Inclusión laboral; discapacidad; mercado de trabajo; Chile.

ABSTRACT This article analyzes the entry into force of Law No. 21.015 which enables people in situations of disability to take part in the labor market . The purpose of this regulation is to improve the employability of people in situations of disabilities in open work environments by reserving $1 \%$ of the 
job quota in companies with 100 or more workers. In this study the limitations of this policy of access to employment for the promotion of the right to work and the configuration of more inclusive societies are examined. According to a critical analysis of the implementation of the legal initiative in the private sector, salary, age and gender gaps are observed in the incorporation and permanence of people in situations of disabilities in the labor market. Finally, it is concluded that labor insertion strategies in the field of disability do not question the power structures in capitalist society and are integrated into the foundations of the neoliberal model in contemporary Chile.

KEYWORDS Labor inclusion; disability; labor market; Chile.

\section{Introducción}

En los últimos años, la inclusión laboral de personas con discapacidad ha ido adquiriendo relevancia en la agenda pública nacional. Este fenómeno se ha asociado a un avance del reconocimiento del derecho al trabajo y la promoción de oportunidades de empleo con base en los planteamientos de la Convención sobre los Derechos de las Personas con Discapacidad (CDPD) de Naciones Unidas, ratificada por el Estado de Chile el año 2008. En nuestro país, la población de adultos con discapacidad (18 años y más), corresponde a un 20\% de la población, un total de 2.606.914 personas (Servicio Nacional de Discapacidad [SENADIS], 2015). El 57,2\% no participa del mercado laboral, lo que corresponde a 1.488 .576 personas. A su vez, los ingresos económicos de las personas con discapacidad son menores a los de las personas que no están bajo esta condición y al total de la población, ingresos que se encuentran por debajo del salario mínimo (SENADIS, 2015). Por otra parte, las bajas tasas de participación laboral afectan de manera diferencial a mujeres con discapacidad, presentando menores tasas de incorporación en el mercado de trabajo.

Con relación al acceso de las personas con discapacidad al mundo del trabajo, el $40 \%$ de las personas con discapacidad están económicamente activas. De las personas que trabajan, un $64,7 \%$ lo realiza de forma asalariada, mientras que un $35,3 \%$ tiene un trabajo independiente. El 5,8\% de las personas con discapacidad que se encuentran trabajando lo hace en el sector público, en contraste con el 51,8\% que lo hace en el sector privado (SENADIS, 2015). De acuerdo con estas cifras, actores institucionales han planteado un creciente interés por la eliminación de las barreras para una participación plena de las personas con discapacidad en la sociedad, impulsando reformas hacia un sistema laboral más inclusivo.

Bajo estas orientaciones, se ha manifestado un giro respecto a la importancia del trabajo en el campo de la discapacidad. Una aproximación tradicional no comprendía el desempleo de las personas con discapacidad como un problema debido a que 
se asumía que no pueden trabajar y deben recibir ayudas por parte del Estado. Esta modalidad centrada en políticas de asistencia y protección social ha transitado hacia un enfoque que comprende la importancia del empleo como vía de integración a la sociedad y acceso a la ciudadanía. En este sentido, la promoción de oportunidades de empleo decente y productivo para personas con discapacidad ha adquirido importancia en los instrumentos internacionales y las legislaciones nacionales (Organización Internacional del Trabajo [OIT], 2015). Al respecto, a nivel global predominan las políticas de inclusión laboral que promueven la presencia de personas con discapacidad en las empresas en base al cumplimiento efectivo de cuotas de reserva.

En Chile, el año 2018 entra en vigencia la Ley No21.015 "Incentiva la inclusión de personas con discapacidad al mundo laboral" que exige la reserva del $1 \%$ de la cuota de empleos para personas con discapacidad en empresas con 100 o más trabajadores. Esta normativa, se ha planteado como una política específica orientada a superar la baja participación de las personas con discapacidad en el mercado laboral, promoviendo su incorporación a los sistemas de trabajo abiertos. En su implementación, se estableció una modalidad gradual para el cumplimiento de la normativa, de acuerdo con el número de trabajadores de las empresas. A su vez, en el ámbito privado, se instauraron medidas de cumplimiento alternativo al proceso de contratación directa, por medio de contratos de prestación de servicios con empresas que tengan contratadas a personas con discapacidad o a través de donaciones a fundaciones relacionadas con el ámbito de la inclusión laboral de personas con discapacidad. De esta manera, la reciente implementación de esta normativa ha implicado un período de acomodación y ajuste de sus reglamentaciones en el escenario laboral chileno, motivo por el que se han realizado limitados estudios sobre su aplicación efectiva.

En este contexto, la presente investigación analiza los alcances de la Ley №21.015 para superar las barreras de entrada y permanencia de las personas con discapacidad en el mundo del trabajo. Para ello, se realiza una reconstrucción conceptual de la relación trabajo y discapacidad, así como una aproximación al panorama del trabajo en el Chile neoliberal. Finalmente, se examinan los alcances y limitaciones de la ley de inclusión laboral en el sector privado, con base en un análisis de cifras oficiales de cumplimiento de esta normativa a dos años de su entrada en vigencia.

\section{Trabajo y discapacidad: barreras de acceso al empleo y gestión de la diversidad en el ámbito laboral}

Las altas tasas de desempleo y la baja inserción laboral de las personas con discapacidad constituyen un fenómeno constante a nivel mundial. Frente a este escenario, la Convención sobre los Derechos de las Personas con Discapacidad (CDPD) de Naciones Unidas ha establecido la importancia de promover políticas y normativas orientadas a favorecer el acceso al empleo, la adquisición de experiencia laboral y el ejercicio del derecho al trabajo de las personas con discapacidad. 
La CDPD establece los principios de no discriminación, participación plena, igualdad de oportunidades y accesibilidad en los contextos de trabajo, enfatizando la importancia de los factores relacionales y contextuales en el ámbito de la discapacidad. Esta perspectiva, se inscribe en el modelo social de la discapacidad, que define la discapacidad como una construcción social (Palacios, 2008). Esta mirada, considera el origen de la discapacidad en la sociedad, pues sería la sociedad quien interpone barreras a la inclusión de las personas al interactuar con su entorno, limitando su participación y ejercicio de derechos en igualdad de condiciones que las demás (Brogna, 2008).

En términos históricos, el modelo social de la discapacidad nace del Movimiento de Vida Independiente y el activismo político de las propias personas con discapacidad, quienes desde la década de los 60' comenzaron a replantearse ciertas estrategias de lucha frente a la situación de opresión social en la que se encontraban inmersas con el fin último de ejercer control sobre sus propias vidas (Barton, 2008). En ese recorrido, la CDPD consolida las reivindicaciones de organizaciones de personas con discapacidad en defensa de sus derechos para participar en la vida comunitaria, dejando atrás el modelo biomédico-rehabilitador y estableciendo estándares internacionales de derechos humanos en el ámbito de la discapacidad de carácter vinculante para los Estados (Cabra, Bariffi \& Palacios, 2007).

En específico, los cambios culturales impulsados por el movimiento de personas con discapacidad y las reformas institucionales promovidas por la CDPD, han contribuido a un examen crítico de la relación trabajo y discapacidad en la actualidad. Como se ha señalado, las personas con discapacidad son un colectivo potencialmente excluido de los espacios de participación social, entre ellos, los entornos laborales. Desde el modelo social de la discapacidad se ha querido dar énfasis a los mecanismos de exclusión que afectan la empleabilidad o la inserción laboral de esta población, así como las barreras actitudinales, los prejuicios y el estigma en torno a la discapacidad.

En este escenario, se ha enfatizado que las personas con discapacidad han sido marginadas de los procesos productivos por sus características individuales, ya que no cumplen con los parámetros y estándares del sistema laboral, según criterios de aptitud laboral que establecen jerarquías entre los sujetos (Barton, 2008). Sin embargo, estas barreras estructurales que sostienen relaciones de desigualdad excluyentes no solo han afectado a las personas con discapacidad sino a diversos grupos sociales que no logran integrarse a los procesos de normalización de la fuerza de trabajo, por lo tanto, no se adaptan a las condiciones laborales impuestas por el sistema capitalista (Barton, 2008).

Para abordar esta problemática, se han desarrollado planes y programas orientados a favorecer la inserción laboral de personas con discapacidad. En el marco de los programas de rehabilitación, predominan los trabajos sin retribución o actividades 
laborales de menor valoración social. En este ámbito han predominado iniciativas de carácter asistencialista en que las personas desarrollan habilidades laborales en entornos cerrados por los cuales reciben un incentivo económico muy bajo con relación al trabajo realizado. Estas modalidades de desarrollo ocupacional, denominadas empleos protegidos, han sido cuestionados ya que no garantizan el derecho al trabajo y sostienen círculos de exclusión del mercado laboral (Chuaqui, 2015). Frente a ello, con el avance del neoliberalismo se han implementado nuevas estrategias para producir sujetos productivos, rentables y eficientes, mediante diversos mecanismos de empleabilidad individual y dinámicas de integración al mercado laboral (Soldatic \& Meekosha, 2012).

Al respecto, en los últimos años han adquirido relevancia la adopción y aplicación de políticas dirigidas a aumentar la empleabilidad de las personas con discapacidad: centros de formación laboral, programas de capacitación laboral, entrenamiento en búsqueda de empleo, estrategias de orientación vocacional, trabajo en prácticas, intermediación laboral, empleos de transición, entre otros. Este conjunto de iniciativas se orienta a superar los problemas de acceso y mantenimiento del empleo en entornos laborales abiertos. En este sentido, el empleo con apoyo se ha planteado como una estrategia efectiva y ampliamente validada para cumplir con dicho objetivo (Vidal, Cornejo \& Arroyo, 2013). Esta iniciativa consiste en la búsqueda y mantenimiento de empleos regulares, estableciendo mecanismos de acompañamiento individualizados, continuos y flexibles en el entorno laboral, así como formación y seguimiento en el puesto de trabajo (Vidal \& Cornejo, 2014).

Con base en estas diversas modalidades de inserción sociolaboral, se ha planteado que el acceso al trabajo asalariado y la obtención de una retribución legalmente regulada para las personas con discapacidad contribuyen a su autonomía económica y a desempeñar un rol activo en la sociedad. Sin embargo, lo anterior ha favorecido la disminución del gasto público y los costos de seguridad social generados por esta comunidad en un contexto neoliberal (Soldatic \& Meekosha, 2012). De esta manera, el análisis de los esfuerzos para superar las barreras de acceso al mercado laboral y garantizar el derecho al trabajo de las personas con discapacidad, debe considerar los aspectos materiales y simbólicos que determinan su conceptualización en el escenario contemporáneo. En ese sentido, a continuación, se plantean los siguientes elementos para un abordaje crítico de la relación trabajo y discapacidad en la actualidad:

- Aproximarse a las barreras de entrada de las personas con discapacidad al mercado de trabajo, en las que adquieren relevancia los estigmas y estereotipos asociados a la discapacidad, así como el nivel de cualificación, la experiencia laboral previa y la red de contactos de las personas con discapacidad que buscan empleo (Cruz, Fadic, Hernández \& Llambías, 2015; Rojas-Carrasco et al., 2019). 
- Indagar en los significados del trabajo para las personas con discapacidad, destacando aspectos como la construcción de identidad y el grado de satisfacción en los entornos laborales, así como sus intereses y motivaciones para integrarse a las dinámicas del empleo formal (Gomes, Carvalho-Freitas \& de Oliveira, 2014; Pereira-Silva, Furtado y Andrade, 2018; Pinto de Lima, Vasconcelos, Brito y Alves, 2013).

- Analizar la relación entre adecuación laboral y mantenimiento del empleo en espacios laborales que garanticen la participación efectiva de la persona con discapacidad en la gestión de la intensidad y ritmo de trabajo (Cea-Madrid, 2021).

- Problematizar las exigencias del ajuste laboral en entornos laborales en los que predomina la estandarización y adaptación de la persona con discapacidad al puesto de trabajo (Cea-Madrid, 2021).

- Considerar las dificultades de las personas con discapacidad para mantenerse laboralmente activas en entornos competitivos en los que predomina el rendimiento y la productividad en base a estándares impuestos (Chuaqui, 2015).

- Reconocer el rechazo de las personas con discapacidad a participar de entornos laborales que no respetan sus singularidades, así como valorar sus cuestionamientos a los procesos de normalización que establece el orden político y económico capitalista (Parker, Blanch, Ordenes \& Navarro, 2020; Pino-Morán \& Rodríguez-Garrido, 2017; Sosa et al., 2015).

- Evaluar el efecto desincentivador de las pensiones de discapacidad y la sobreprotección familiar que refuerzan el sostenimiento de relaciones de dependencia y dinámicas de institucionalización en el campo de la discapacidad (Castillo-Parada \& Cea-Madrid, 2017).

Todos estos ámbitos de indagación escapan ampliamente a las políticas de integración laboral que se han implementado en el último tiempo en nuestro país. Por una parte, en el ámbito empresarial, se han reforzado estrategias de sensibilización para considerar postulantes con discapacidad en los procesos de reclutamiento y selección indicando los siguientes beneficios: aumento de la productividad, menores índices de rotación y ausentismo, mejoramiento del clima laboral, mayor reputación para la organización y aumento del valor para los accionistas (Organización Internacional del Trabajo, 2013; Fundación Descúbreme, 2019). De esta manera, el criterio económico de mayor rentabilidad y mejor competitividad de las empresas en el mercado se constituye como un estímulo relevante para la integración de personas con discapacidad en el ámbito laboral.

Por otra parte, las vías alternativas de cumplimiento de la Ley № 21.015 reúnen a un conjunto de fundaciones con programas de inserción laboral que contemplan una heterogeneidad de enfoques y perspectivas. Al respecto, se encuentran organizaciones que desarrollan programas de capacitación y prácticas laborales en entornos 
protectores, en los que predominan indicadores de productividad y mecanismos de evaluación de desempeño para la asignación de incentivos muy bajos con relación al trabajo realizado. A su vez, otras organizaciones promueven el ajuste de la persona con discapacidad en entornos laborales competitivos y con retribución de mercado, sin embargo, reproducen modelos de normalización y adaptación social en los puestos de trabajo. De acuerdo con estas prácticas, en el escenario nacional predominan las modalidades de empleo protegido y empleo con apoyo en la obtención de recursos vía donaciones.

Finalmente, a nivel local se han impulsado programas de ayuda para el autoempleo de personas con discapacidad bajo un modelo cooperativo. Estos programas de apoyo estatal se han orientado a financiar microemprendimientos con el fin de estimular la participación laboral de personas con discapacidad para la conformación de "cooperativas inclusivas" (Westermeier, Salas, Jaramillo \& Torres, 2018). Sin embargo, estas unidades económicas se insertan en dinámicas de competitividad para la obtención de fondos concursables, su sustentabilidad se basa en relaciones de dependencia a subsidios estatales y su modelo de gestión se centra en la continua supervisión de profesionales que anulan su autonomía productiva.

En definitiva, el conjunto de iniciativas orientadas a promover la participación laboral de personas con discapacidad ha desestimado el desarrollo de perspectivas críticas y alternativas en torno a la relación trabajo y discapacidad, enfatizando una dependencia y subordinación a las dinámicas del mercado capitalista. Para comprender esta ausencia de debate, es necesario examinar los alcances de la hegemonía neoliberal en el mundo del trabajo en nuestro país.

\section{Trabajo y neoliberalismo: flexibilidad laboral y precarización del empleo en el Chile contemporáneo}

En el contexto europeo, las leyes que reservan cuotas laborales para personas con discapacidad entran en su apogeo en el contexto de un capitalismo financiero y globalizado (Harvey, 2007). Lo anterior, se ha asociado a una extensión de las lógicas del mercado en la sociedad y al desmantelamiento del Estado social como agente integrador. Al respecto, se ha planteado que la inseguridad social es una de las consecuencias masivas de ese deterioro de las protecciones colectivas (Castel, 2006; 2010). En este marco, los grupos sociales en situación de asistencia y vulnerabilidad han sido los más afectados con los recortes en políticas sociales y medidas de ajuste del gasto social (Arenas-Conejo \& Pié-Balaguer, 2014; Muyor-Rodríguez, 2018).

Desde una aproximación histórica, Chile se erigió durante Dictadura cívico-militar de Augusto Pinochet como laboratorio del neoliberalismo con base en el desmantelamiento de las estructuras de derechos sociales como la educación, la salud y la seguridad social. En particular, el Plan Laboral de 1979 se ha constituido como la 
piedra angular de las transformaciones neoliberales en el mundo del trabajo (RuizBruzzone, 2016). Bajo estas orientaciones, el régimen neoliberal ha establecido en nuestro país mecanismos de expropiación financiera y desposesión salarial que han agudizado las desigualdades en el mundo del trabajo (Narbona \& Páez, 2014).

En Chile, investigaciones recientes en el ámbito del trabajo dan cuenta que el mercado laboral se define por un aumento de los mecanismos de precariedad y flexibilización del empleo (Blanco \& Julián, 2019; Julián, 2014; Julián \& Galliorio, 2018). El panorama del trabajo se caracteriza por extensas jornadas laborales, bajos salarios y altas tasas de endeudamiento (Stecher \& Sisto, 2019). A su vez, en un escenario de ausencia de derechos laborales colectivos, los marcos normativos limitan la organización de los trabajadores y la acción sindical (Julián \& Galliorio, 2018; Ratto, 2019). Junto con ello, los mecanismos de desregulación del mercado de trabajo han sostenido cambios profundos en las relaciones de dependencia salarial. Estas transformaciones, ubican a amplios sectores de la población en condiciones de desprotección social e inestabilidad laboral. En particular, la conformación de un mercado de trabajo bajo las coordenadas del rendimiento y la competitividad destinan a los grupos sociales más desfavorecidos a la pobreza, el desempleo y la exclusión social (Stecher \& Godoy, 2014).

Con relación al modelo productivo y la estructura ocupacional en Chile, el panorama del trabajo se caracteriza por una predominancia de los servicios y el comercio, estableciéndose el sector terciario de la economía como principal ámbito de empleabilidad (Ruiz \& Boccardo, 2014). Junto con ello, las particularidades del modelo empresarial chileno han determinado un desarrollo creciente de la externalización de servicios y el aumento de la subcontratación (Julián \& Galliorio, 2018). En este sentido, en los modelos de integración al mercado productivo las formas de trabajo adquieren un carácter inestable, con un alto nivel de rotación laboral y extensas condiciones de flexibilidad contractual (Stecher \& Sisto, 2019). Finalmente, el ordenamiento descrito se complementa con un amplio sector informal, que reúne a un conjunto de trabajadores y trabajadoras no calificadas insertas en sectores de baja productividad fuera de los marcos legales de regulación del empleo (Ruiz \& Boccardo, 2014).

En este marco, encontramos que las tasas de desempleo y las cifras de empleo de menor estabilidad tienen relación con el aumento de las condiciones de precariedad en el conjunto de la clase trabajadora. En un escenario en que el trabajo formal se hace más escaso y el combate al desempleo parece encontrarse con una barrera estructural, la implementación de la Ley $\mathrm{N}^{\circ} 21.015$ se erige como una pieza clave de análisis del mercado laboral chileno. La normativa pretende eliminar brechas de acceso y garantizar una cuota efectiva de participación en el mercado laboral para un sector de la población como son las personas con discapacidad, con el propósito que 
transiten desde una situación de no trabajo (desocupados) a una situación de trabajo dependiente (asalariados). De esta manera, esta legislación se orienta a la producción de sujetos laborales con el objetivo de reconfigurar el lugar tradicional de las personas con discapacidad como individuos improductivos e incapaces (Ñúnez-Parra, 2020).

Junto con ello, la Ley № 21.015 se inscribe en la agenda de desarrollo sostenible al contribuir a la inclusión y promover una mayor participación laboral de las personas con discapacidad, superando las barreras que sustentan la exclusión de este colectivo de la economía formal y oficial (Montes, 2020). Por lo tanto, la normativa pretende superar las desigualdades que sitúan a las personas con discapacidad en el sector informal del trabajo por cuenta propia, así como subvertir el vínculo entre discapacidad y pobreza, así como otras condiciones de vulnerabilidad y desafiliación que alejan a este colectivo de la prosperidad económica. En este sentido, se ha planteado el lugar central del trabajo para superar imaginarios negativos en torno a la discapacidad, los que se han construido según nociones de carencia e impedimento, como es la ausencia de ingresos económicos o la falta de empleo. No obstante, esta apuesta de desarrollo inclusivo se basa en la confianza de que el mercado de trabajo asigna los ingresos de manera justa e igualitaria, y que es eficiente para asignar oportunidades y distribuir salarios.

Por otra parte, la Ley $\mathrm{N}^{\circ} 21.015$ tiene por finalidad modernizar la legislación laboral, buscando establecer jornadas de trabajo adaptables que fomenten la conciliación trabajo y familia y mejoren la calidad de vida, así como garantizar un mercado laboral que dignifique a las personas (Montes, 2020). Sin embargo, cabe resaltar que esta realidad no ha sido posible para el conjunto de los trabajadores y trabajadoras, quienes poseen una escasa capacidad de maniobra para mejorar sus salarios y negociar sus condiciones de trabajo, por lo tanto, la influencia de un sector minoritario de la fuerza de trabajo como son las personas con discapacidad para promover contextos laborales inclusivos son limitadas en un contexto neoliberal en que predominan los intereses del poder empresarial (Julián \& Galliorio, 2018; Ruiz \& Boccardo, 2014).

De esta manera, la creación de legislaciones y sus respectivas reglamentaciones que no contemplan un cuestionamiento a las estructuras de poder que configuran el sistema capitalista se muestran insuficientes para garantizar la igualdad de oportunidades de empleo y el derecho al trabajo en el campo de la discapacidad. Bajo esta aproximación crítica, cabe problematizar el acceso y permanencia en el mercado de trabajo de las personas con discapacidad con base en políticas de integración laboral que se desarrollan bajo el neoliberalismo. En este sentido, a continuación, se analizarán los alcances y limitaciones de la Ley № 21.015 en el sector privado a dos años de su entrada en vigencia. 


\section{Metodología}

En Chile, la Ley No 21.015 "Incentiva la inclusión de personas con discapacidad al mundo laboral" ha determinado que a contar del 1 de abril de 2018 las empresas de 200 o más trabajadores deben reservar un $1 \%$ de su planilla para personas con discapacidad. A su vez, desde el 1 de abril de 2019, las empresas que cuentan con 100 a 199 trabajadores están obligadas al cumplimiento de esta normativa. Sin embargo, la legislación establece medidas de cumplimiento alternativas a las empresas que no puedan efectuar total o parcialmente la obligación del 1\%. La normativa contempla dos modalidades de ejecución alternativa:

a) Realizar donaciones en dinero a organizaciones acreditadas como donatarias y que tengan proyectos aprobados mediante resolución por el Ministerio de Desarrollo Social (MIDESO), lo que las faculta para recibir donaciones para los fines de esta ley. Respecto al monto anual de las donaciones, este no podrá ser inferior a 24 ingresos mínimos mensuales por persona no contratada. Cabe señalar que estas donaciones no darán derecho a crédito o beneficios tributarios y tendrán la calidad de gasto necesario o aceptado para las empresas.

b) Celebrar contratos de prestación de servicios con empresas que tengan personas con discapacidad contratadas en su plantilla, las que deben prestar servicios en la empresa contratante, afectada por la obligación legal de cumplimiento del 1\%. Con relación al monto anual de los contratos celebrados, estos no podrán ser inferiores a 24 ingresos mínimos mensuales respecto de cada trabajador que debía ser contratado directamente por la empresa.

En estos términos, para aproximarse a las condiciones de cumplimiento de la Ley No 21.015, en junio del 2020 a través del portal de acceso a la información pública se solicitó a la Dirección del Trabajo - entidad dependiente del Ministerio del Trabajo y Previsión Social encargada de orientar la correcta aplicación de esta normativa, ejercer un rol fiscalizador y realizar el registro electrónico de los contratos de trabajo y/o las medidas alternativas implementadas por los empleadores - información sobre el número total de trabajadores(as) contratados(as) bajo la ley de inclusión laboral, las condiciones de contratación y las medidas alternativas en que las empresas dieron cumplimiento a esta normativa.

De manera complementaria a la información remitida por este organismo, se realizó una pesquisa en fuentes oficiales del Ministerio de Desarrollo Social del catastro de las asociaciones, corporaciones o fundaciones cuyo objeto social incluya la capacitación, rehabilitación, promoción y fomento para la creación de empleos, contratación o inserción laboral de personas con discapacidad que se encuentran inscritas en el registro de donatarios bajo la Ley $\mathrm{N}^{\circ} 19.885$ de Donaciones con Fines Sociales, que han postulado iniciativas al Banco de Proyectos de la Ley $\mathrm{N}^{\circ} 21.015$ y han sido aprobadas entre los años 2018 y 2019. 
De acuerdo con los datos obtenidos, a continuación, se presentan los indicadores referidos a las medidas de cumplimiento de la Ley No 21.015 con base en la contratación directa de personas con discapacidad y en las modalidades subsidiarias de cumplimiento en el ámbito privado en el período 2018 - 2020.

\section{Implementación de la Ley $\mathbf{N}^{\circ} 21.015$ en el sector privado: de la subordinación asalariada a la caridad neoliberal}

Con base en la información recopilada sobre el total de empresas y trabajadores(as) con contratos registrados ante la Dirección del Trabajo, en la tabla 1 se observa una disminución del número de empresas y trabajadores(as) que registran su actividad desde la entrada en vigencia de la Ley $\mathrm{N}^{\circ} 21.015$ a mayo del 2020. Por otra parte, se observa una brecha de género en las personas con relación laboral vigente en la medida que el número de trabajadores hombres duplica al número de trabajadoras mujeres. Finalmente, cabe destacar que cerca de la mitad de los(as) trabajadores(as) con relación laboral vigente $(46,5 \%)$ iniciaron su contratación antes de la entrada en vigencia de la normativa.

Tabla 1. Cantidad de empresas y trabajadores con contratos registrados bajo Ley 21.015 ante la Dirección del Trabajo al 31/05/2020.

\begin{tabular}{|c|c|c|c|c|c|}
\hline \multirow[t]{8}{*}{ Trabajadores } & Desde la & \multicolumn{4}{|c|}{ Con relación laboral vigente al 31/05/2020 } \\
\hline & \multirow[t]{7}{*}{23.064} & \multirow[t]{7}{*}{19.488} & \multirow{3}{*}{$\begin{array}{c}\text { Fecha de } \\
\text { inicio } \\
\text { relación } \\
\text { laboral }\end{array}$} & Antes del 15/06/2017 & 9.047 \\
\hline & & & & $\begin{array}{c}\text { A partir } \\
\text { del } 15 / 06 / 2017\end{array}$ & 10.410 \\
\hline & & & & Sin información & 31 \\
\hline & & & \multirow[t]{2}{*}{ Sexo } & Hombres & 12.541 \\
\hline & & & & Mujeres & 6.947 \\
\hline & & & \multirow[t]{2}{*}{ Condición } & $\begin{array}{c}\text { Asignatarios de } \\
\text { pensión de invalidez }\end{array}$ & 3.380 \\
\hline & & & & $\begin{array}{l}\text { Personas con } \\
\text { discapacidad }\end{array}$ & 16.108 \\
\hline Empresas & 4.085 & 3.829 & & & \\
\hline
\end{tabular}

Fuente: Departamento de Estudios, Dirección del Trabajo. 
Con relación a los contratos registrados bajo la Ley de Inclusión Laboral que no se encuentran vigentes a mayo del 2020, en la tabla 2 se muestra que las principales causas de término de contrato se asocian a relaciones laborales que tenían un plazo fijo de término y en las cuales el trabajador decide renunciar. Otros motivos se relacionan con el término de contrato por parte de la empresa y el abandono del puesto de trabajo por parte de las personas con discapacidad, motivos que explican el 79,9\% de las causas de término de contrato.

Tabla 2. Cantidad y porcentaje de contratos no vigentes registrados bajo la ley de inclusión ante la Dirección del trabajo, según causal del término de contrato al 31/05/2020.

\begin{tabular}{|l|c|c|}
\hline Causal del término de contrato & $\begin{array}{c}\text { Cantidad de } \\
\text { contratos } \\
\text { terminados }\end{array}$ & Porcentaje \\
\hline Vencimiento del plazo convenido en el contrato & 1.511 & $26,1 \%$ \\
\hline $\begin{array}{l}\text { Renuncia del trabajador, dando aviso a su empleador } \\
\text { con treinta días de anticipación, a lo menos }\end{array}$ & 1.430 & $24,7 \%$ \\
\hline Necesidad de la empresa, establecimiento o servicio & 972 & $16,8 \%$ \\
\hline No concurrencia a sus labores sin causa & 713 & $12,3 \%$ \\
\hline Conclusión del trabajo que dio origen al contrato & 569 & $9,8 \%$ \\
\hline Otros motivos & 588 & 10,3 \\
\hline Total & 5.783 & $100 \%$ \\
\hline
\end{tabular}

Fuente: Elaboración propia en base a datos obtenidos del Departamento de Estudios, Dirección del Trabajo.

Respecto a la duración de los contratos que han dado término a la relación laboral a mayo del 2020, en la tabla 3 se muestra que la mayoría de los contratos tuvo una duración de 1 hasta 6 meses, lo que destaca el bajo período de tiempo que se sostuvo la relación laboral para las personas con discapacidad. Por otra parte, datos complementarios indican que un $73,6 \%$ de los contratos terminados correspondían a contratos de jornada completa y un 47,92 representaban un contrato de plazo fijo, por lo tanto, la mayoría de las personas con discapacidad contratadas tenían un solo trabajo y alrededor de la mitad del total tenían un contrato con fecha de caducidad establecida de antemano al inicio de la relación laboral. 
Tabla 3. Cantidad y porcentaje de contratos terminados registrados bajo la ley de inclusión ante la Dirección del trabajo, según antigüedad del contrato al término de la relación laboral al 31/05/2020.

\begin{tabular}{|l|c|c|}
\hline Antigüedad del contrato & Cantidad de contratos & Porcentaje \\
\hline Hasta 1 mes (30 días) & 756 & $13,1 \%$ \\
\hline De más de 1 hasta 6 meses (31 a 180 días) & 2.417 & $41,8 \%$ \\
\hline De más de 6 meses hasta un 1 año (181 a 365 días) & 849 & $14,7 \%$ \\
\hline De más de 1 hasta 3 años (366 a 1.095 días) & 746 & $12,9 \%$ \\
\hline De más de 3 años & 982 & $17 \%$ \\
\hline Sin información & 33 & $0,6 \%$ \\
\hline Total & 5.783 & \\
\hline
\end{tabular}

Fuente: Elaboración propia en base a datos obtenidos del Departamento de Estudios, Dirección del Trabajo.

Respecto a los contratos actualmente vigentes para la Dirección del Trabajo, en la tabla 4 se observa que constituyen modalidades de contratación que han sido más prolongadas en el tiempo. Por ejemplo, un $45,1 \%$ de los contratos vigentes han tenido una duración de 3 años o más, lo que demuestra que las personas con discapacidad han sido contratadas antes de la entrada en vigencia de la Ley $\mathrm{N}^{\circ} 21.015$. Junto con ello, datos complementarios indican que un $75,6 \%$ de los contratos vigentes corresponden a contratos de jornada completa (31 o más horas semanales) y un 70,6\% corresponde a una relación laboral de contrato indefinido. De esta manera, encontramos un grupo significativo de personas con discapacidad que presentan contratos estables que han logrado acceder al mercado de trabajo desde antes de la implementación de la Ley $\mathrm{N}^{\circ}$ 21.015 como se observa en la tabla 5 .

Tabla 4. Cantidad y porcentaje de contratos vigentes registrados bajo la ley de inclusión ante la Dirección del Trabajo, según antigüedad del contrato al 31/05/2020.

\begin{tabular}{|l|c|c|}
\hline Antigüedad del contrato & Cantidad de contratos & Porcentaje \\
\hline Menos de 6 meses & 1.396 & $6,9 \%$ \\
\hline De más de 6 meses hasta 1 año (181 a 365 días) & 2.287 & $11,3 \%$ \\
\hline De más de 1 hasta 3 años (366 a 1.095 días) & 7.388 & $36,5 \%$ \\
\hline De más de 3 hasta 5 años (1.096 a 1.825 días) & 1.984 & $9,8 \%$ \\
\hline De más de 5 hasta 10 años (1.826 a 3.650 días) & 3.166 & $15,6 \%$ \\
\hline Más de 10 años (Más de 3.650 días) & 3.993 & $19,7 \%$ \\
\hline Sin información & 31 & 0,2 \\
\hline Total & 20.245 & $100 \%$ \\
\hline
\end{tabular}

Fuente: Departamento de Estudios, Dirección del Trabajo. 
Tabla 5. Cantidad y porcentaje de contratos vigentes registrados bajo la ley de inclusión ante la Dirección del Trabajo, según fecha de inicio de la relación laboral al $31 / 05 / 2020$.

\begin{tabular}{|l|c|c|}
\hline Fecha de inicio de la relación laboral & Cantidad de contratos & Porcentaje \\
\hline Antes del 01/04/2018 & 11.020 & $54,4 \%$ \\
\hline A partir del 01/04/2018 & 9.194 & $45,4 \%$ \\
\hline Sin información & 31 & $0,2 \%$ \\
\hline Total & 20.245 & $100 \%$ \\
\hline
\end{tabular}

Fuente: Departamento de Estudios, Dirección del Trabajo.

Por otra parte, en la tabla 6 se constata que un 59,9\% de los(as) trabajadores(as) con discapacidad que presentan contratos vigentes tienen 41 años o más, por lo tanto, las personas que se encuentran en la adultez media han establecido formas de contratación más duraderas y con mayor estabilidad laboral. Esta perspectiva, confronta con el bajo nivel de participación laboral del tramo etario de las personas con discapacidad que inician su carrera laboral, evidenciando brechas de acceso para las personas más jóvenes en las modalidades de contratación actualmente vigentes.

Tabla 6. Cantidad y porcentaje de trabajadores contratados bajo ley de inclusión laboral, con contratos vigentes registrados ante la Dirección del Trabajo, según tramo etario al 31/05/2020.

\begin{tabular}{|l|c|c|}
\hline Tramo Etario & Cantidad de trabajadores & Porcentaje \\
\hline Menos de 18 años & 4 & 0,0 \\
\hline 18 a 25 años & 1.272 & $6,5 \%$ \\
\hline 26 a 40 años & 6.501 & $33,4 \%$ \\
\hline 41 a 59 años & 8.439 & $43,3 \%$ \\
\hline 60 años o más & 3.230 & $16,6 \%$ \\
\hline Sin fecha de nacimiento reportada & 42 & $0,2 \%$ \\
\hline Total & 19.488 & $100 \%$ \\
\hline
\end{tabular}

Fuente: Departamento de Estudios, Dirección del Trabajo.

Conforme a lo anteriormente descrito, en la tabla 7 se muestran los montos de remuneraciones obtenidas por las personas con discapacidad con contrato vigente. Sobre estas cifras, cabe destacar la brecha salarial entre hombres y mujeres y que, además, la remuneración promedio mensual se observa levemente más baja con relación al ingreso laboral promedio de la población. 
Tabla 7. Remuneración imponible promedio y mediana mensual de trabajadores(as) contratados bajo ley de inclusión laboral, con contratos vigentes registrados ante la Dirección del Trabajo y cantidad de trabajadores, según sexo de los trabajadores al $31 / 05 / 2020$.

\begin{tabular}{|l|l|l|l|}
\hline Sexo & Monto promedio & Monto mediana & Cantidad de trabajadores \\
\hline Hombres & $\$ 587.580,17$ & $\$ 457.480,00$ & 11.260 \\
\hline Mujeres & $\$ 528.816,29$ & $\$ 420.000,00$ & 6.255 \\
\hline Total & $\$ 566.594,27$ & $\$ 445.000,00$ & 17.515 \\
\hline
\end{tabular}

Fuente: Departamento de Estudios, Dirección del Trabajo.

Con relación al cumplimiento de la Ley $\mathrm{N}^{\circ} 21.015$ a través de medidas alternativas, en la tabla 8 se evidencia una disminución en el número de empresas que realizan actividades de contratación de servicios con empresas que cumplen con la cuota inclusiva, así como en el número de trabajadores beneficiados con esta modalidad. Por el contrario, se observa un aumento del número de empresas que efectúan donaciones a fundaciones del mundo de la discapacidad y una ampliación del número de fundaciones beneficiadas con esta medida alternativa para dar cumplimiento a la normativa. Cabe señalar que, al 31 de diciembre de cada año, las empresas de más 100 trabajadores que no han contratado el $1 \%$ de personas con discapacidad pueden utilizar el cumplimiento alternativo de donación para no ser multadas.

Tabla 8. Cantidad de empresas que han informado a la Dirección del Trabajo, cumplir la Ley de Inclusión Laboral por medio de medidas alternativas, según año de declaración al 31/05/2020.

\begin{tabular}{|c|c|c|c|}
\hline $\begin{array}{l}\text { Año de la } \\
\text { declaración }\end{array}$ & $\begin{array}{l}\text { Medida } \\
\text { alternativa }\end{array}$ & $\begin{array}{l}\text { Cantidad de } \\
\text { empresas }\end{array}$ & \\
\hline \multirow[t]{4}{*}{2019} & \multirow{2}{*}{$\begin{array}{l}\text { Contratación } \\
\text { de servicios }\end{array}$} & \multirow[t]{2}{*}{230} & Trabajadores contratados por EST \\
\hline & & & 1.001 \\
\hline & \multirow[t]{2}{*}{ Donaciones } & \multirow[t]{2}{*}{268} & $\begin{array}{l}\text { Fundaciones/proyectos que han recibido } \\
\text { donaciones por Ley de Inclusión }\end{array}$ \\
\hline & & & 15 \\
\hline \multirow[t]{4}{*}{2020} & \multirow{2}{*}{$\begin{array}{l}\text { Contratación } \\
\text { de servicios }\end{array}$} & \multirow[t]{2}{*}{183} & Trabajadores contratados por EST \\
\hline & & & 788 \\
\hline & \multirow[t]{2}{*}{ Donaciones } & \multirow[t]{2}{*}{328} & $\begin{array}{l}\text { Fundaciones/proyectos que han recibido } \\
\text { donaciones por Ley de Inclusión }\end{array}$ \\
\hline & & & 24 \\
\hline
\end{tabular}

Fuente: Departamento de Estudios, Dirección del Trabajo. 
Tabla 9. Fundaciones beneficiadas y montos de donaciones recibidas por proyectos asociados a la Ley 21.015 (2018-2019).

\begin{tabular}{|c|c|}
\hline Institución & $\begin{array}{l}\text { Monto } \\
\text { (pesos chilenos) }\end{array}$ \\
\hline Fundación ConTrabajo & 5.224 .120 .000 \\
\hline Fundación TACAL & 3.598 .155 .004 \\
\hline Fundación AVANZA Inclusión Socio-Laboral & 3.338.417.500 \\
\hline Fundación Descúbreme & 2.470.293.280 \\
\hline Fundación Luz (Sociedad Protectora de Ciegos Santa Lucía) & 1.746 .042 .040 \\
\hline ONG Casa de Acogida la Esperanza & 1.511.417.250 \\
\hline Fundación de Beneficencia Ayuda y Esperanza-Emplea & 1.392 .971 .417 \\
\hline Fundación para Ayuda y Rehabilitación de Discapacitados & 1.079 .266 .000 \\
\hline Fundación CpueD & 1.006 .842 .256 \\
\hline Corporación Apertura para el Mañana & 923.770 .556 \\
\hline ONG Instituto de Rehabilitación de Valparaíso & 859.120 .000 \\
\hline ONG Pather Nostrum & 844.731 .845 \\
\hline Fundación Arando Esperanza & 723.714 .502 \\
\hline Fundación Lucha contra la Retinitis Pigmentosa & 674.310 .000 \\
\hline Fundación Chilena para el Síndrome de Down "Complementa" & 629.909 .395 \\
\hline Corporación Respétame & 491.689 .376 \\
\hline Fundación Amigos de Jesús & 472.266 .000 \\
\hline Fundación Best Buddies Chile & 441.240 .620 \\
\hline $\begin{array}{l}\text { Fundación Nacional para la Accesibilidad, el Diseño Universal y la In- } \\
\text { clusión Social }\end{array}$ & 411.931 .332 \\
\hline Fundación Incluir & 376.227 .000 \\
\hline Fundación Amigos Por Siempre & 318.661 .120 \\
\hline Fundación Avanzar Juntos & 208.167 .015 \\
\hline $\begin{array}{l}\text { ONG de Desarrollo Corporación de Educación y Salud para el Sín- } \\
\text { drome de Down }\end{array}$ & 176.600 .000 \\
\hline Corporación de Padres y Amigos de Personas con Déficit & 148.955 .200 \\
\hline Corporación de Ayuda al Limitado Visual & 50.150 .000 \\
\hline Total & 29.118 .968 .708 \\
\hline
\end{tabular}

Fuente: elaboración propia en base a datos obtenidos desde el Ministerio de Desarrollo Social, Gobierno de Chile. 
Finalmente, sobre las fundaciones que han recibido donaciones como medida alternativa de cumplimiento en los años 2018-2019, se registran 40 proyectos beneficiados bajo un monto total de inversión de $\$ 29.118 .968 .708$. En la tabla 9, se observa que solo 4 fundaciones reciben el 50,25\% de los montos asociados a donaciones en el período analizado. Al respecto, estas fundaciones dan cuenta de una multiplicidad de enfoques, población beneficiaria y cobertura territorial en el desarrollo de sus proyectos. Las características de sus iniciativas se describen a continuación: programa de apoyo a la permanencia laboral e inclusión social de personas con discapacidad cognitiva con base en un abordaje sistémico de intermediación laboral (Fundación ConTrabajo), un nuevo centro de formación para el trabajo y programas de formación y capacitación laboral para personas con Discapacidad Moderada a Severa (Fundación TACAL), programas de inclusión sociolaboral, exploración vocacional y colocación laboral a través del modelo de empleo con apoyo (Fundación AVANZA Inclusión Socio-Laboral), así como un programa de capacitación en oficios básicos e inclusión para personas con discapacidad cognitiva (Fundación Descúbreme).

A partir de estos resultados, se ha descrito un panorama global de la implementación de la Ley $\mathrm{N}^{\circ} 21.015$ a dos años de su entrada en vigencia a nivel nacional, estableciendo sus principales ámbitos de desarrollo y características centrales que permiten configurar una evaluación crítica de su aplicación en el sector privado.

\section{Consideraciones finales}

Los alcances de la Ley $\mathrm{N}^{\circ} 21.015$ "Incentiva la inclusión de personas con discapacidad al mundo laboral" dan cuenta de sus limitaciones para promover el acceso al mercado laboral y garantizar el derecho al trabajo en el campo de la discapacidad. Si bien la normativa presenta restricciones en su alcance debido a la baja cuota de incorporación laboral definida en la regulación ( $1 \%$ de la plantilla), los aspectos más sensibles de su aplicación refieren a la generalidad de los procesos de contratación que no han contribuido a aumentar de manera significativa la empleabilidad de las personas con discapacidad desde su entrada en vigencia. En ese recorrido, la falta de incorporación de ajustes razonables y medidas de apoyo para la inclusión laboral de acuerdo con los estándares internacionales de derechos humanos permite comprender la brecha observada entre personas con discapacidad con un alto nivel de integración laboral y otras que no logran insertarse en puestos y procesos laborales a largo plazo.

En concreto, las principales restricciones de la normativa refieren a que cerca de la mitad de las personas con discapacidad contratadas obtuvieron su trabajo antes de la promulgación de la Ley $\mathrm{N}^{\circ} 21.015$, lo que demuestra que esta legislación no ha implicado un impulso sostenido de la contratación de personas con esta condición a dos años de su implementación. Por otra parte, no ha logrado subvertir las inequidades que afectan de manera diferencial a mujeres y personas jóvenes con discapacidad 
ya que presentan menores tasas de participación laboral a dos años de la entrada en vigencia de la normativa. A su vez, las cifras de inclusión de las personas con discapacidad en el mundo del trabajo dan cuenta de una baja calidad laboral, representada por la temporalidad de los contratos y la obtención de puestos de menor salario con relación al ingreso promedio nacional. De esta manera, la legislación no ha logrado superar las brechas salariales, etarias y de género que caracterizan el panorama laboral de la discapacidad en Chile.

En este sentido, es posible sostener que la Ley $\mathrm{N}^{\circ} 21.015$ se inscribe en los elementos transversales que caracterizan una estructura laboral estratificada que afecta de manera diferencial a determinados grupos sociales, con mercados laborales segregados que instauran la exclusión de personas con discapacidad del trabajo remunerado. Al respecto, las cifras presentadas en este estudio tienen su reflejo en el mercado laboral chileno instaurado bajo el neoliberalismo, en términos de temporalidad de los contratos, las bajas remuneraciones y la reproducción de una brecha salarial asociada a la condición de género y nivel educativo (Ruiz \& Boccardo, 2014; Stecher \& Sisto, 2019). Por lo tanto, estas barreras impactan en la baja incorporación y permanencia laboral de las personas con discapacidad en el mundo del trabajo y no contribuyen al desarrollo de un modelo de inclusión laboral sostenible.

Sin embargo, el neoliberalismo no solo ha afectado las relaciones laborales, también se ha asociado a la expansión de la inversión empresarial y la mercantilización de nuevas áreas de la vida social (Harvey, 2007). Con relación a las medidas alternativas de cumplimiento de la Ley $\mathrm{N}^{\circ} 21.015$, cabe destacar que se observa una pluralidad de instituciones privadas que han conseguido beneficios económicos en el marco de la normativa. Lo anterior, ha tenido un impulso con base en esta nueva regulación adquiriendo en este caso las corporaciones de derecho privado y organizaciones no gubernamentales vinculadas a la inclusión laboral de personas con discapacidad no solo subsidios y fondos del Estado, sino también donaciones de empresas privadas como mecanismo de reemplazo a la contratación directa de este colectivo. Esto ha contribuido a una expansión de estas iniciativas con base en heterogéneas modalidades de integración laboral y a un aumento de la competitividad entre organismos privados para la obtención de beneficios, limitando precisamente el cumplimiento de los objetivos de la Ley de inclusión laboral en términos de la inserción de las personas con discapacidad en el ámbito del empleo ordinario. De esta manera, el derecho al trabajo es reemplazado por iniciativas privadas que obtienen utilidades prestando servicios para volver rentables y productivas a las personas con discapacidad, logrando insertarlas a la lógica capitalista de maximización de ganancias.

En consecuencia, la Ley $\mathrm{N}^{\circ} 21.015$ al establecer las disposiciones reglamentarias que posibilitan la entrega de donaciones en dinero por parte de las empresas privadas a fundaciones, contribuye a mantener inalteradas las brechas sociales y las barreras 
de acceso para la contratación directa de personas con discapacidad, afectando la integración laboral e igualdad de oportunidades plena de este colectivo. A su vez, estos mecanismos de cumplimiento alternativo limitan los alcances de la accesibilidad en el mundo del trabajo bajo los principios de igualdad y no discriminación, más bien han posibilitado una extensión de las prácticas asistencialistas para la inserción laboral de las personas con discapacidad en entornos laborales abiertos o protegidos que refuerzan dinámicas de dependencia, pasividad e invisibilidad social. En efecto, las disposiciones de cumplimiento alternativo de la normativa han estimulado renovadas prácticas de caridad en el ámbito de la discapacidad.

En síntesis, la Ley $\mathrm{N}^{\circ} 21.015$ entronca con los patrones estructurales de subordinación del mundo del trabajo bajo el neoliberalismo y se inscribe en la tradición de caridad que caracteriza al campo de la discapacidad en Chile (Ferrante, 2017; Humeres, 2019). Si bien se han presentado datos relevantes en torno a la implementación de esta normativa, es necesario observar las zonas grises en las que no ha sido posible visualizar con claridad los alcances de esta política de inclusión laboral. En particular, en futuras investigaciones sería relevante indagar en los programas de inserción laboral de las fundaciones que han recibido donaciones; también abordar los alcances de la normativa en las áreas de recursos humanos, gestión de personas y organización sindical luego de la publicación de la Ley № 21.275 que modifica el Código del Trabajo y obliga a las empresas a contratar a una persona con conocimientos específicos en materias que fomenten la inclusión laboral de personas con discapacidad, implementar políticas de inclusión y capacitar en la materia a todo el personal. Por otra parte, aproximarse a los sentidos en torno al trabajo y los significados sobre la inclusión laboral en personas con discapacidad, indagando en los facilitadores y obstaculizadores presentes en el proceso. Finalmente, considerar alternativas a la incorporación de las personas con discapacidad al trabajo asalariado con base en iniciativas que se orienten a cuestionar las estructuras del modelo neoliberal y los valores que lo sostienen. $\mathrm{Al}$ respecto, el modelo cooperativo basado en la participación democrática en la toma de decisiones y la distribución igualitaria de los recursos ha contribuido a promover la autonomía de las personas con discapacidad para decidir y elegir libremente sus fuentes de trabajo, así como a promover el desarrollo de entornos laborales inclusivos y accesibles (Cea-Madrid, 2021). En este sentido, el acceso al empleo de las personas con discapacidad y la implementación de modelos alternativos de inclusión laboral, constituyen un desafío para garantizar el derecho al trabajo, y a su vez, una agenda promisoria de investigación en el Chile contemporáneo. 


\section{Referencias}

Arenas-Conejo, Miriam \& Asun Pié-Balaguer (2014). Las comisiones de diversidad funcional en el 15M español: poner el cuerpo en el espacio público. Política y Sociedad, 51(1): 227-245. Doi: 10.5209/rev_POSO.2014.v51.n1.42459.

Barton, Len (Comp.) (2008). Superar las barreras de la discapacidad. Morata.

Blanco, Osvaldo \& Julián Dasten (2019). Una tipología de precariedad laboral para Chile: La precariedad como fenómeno transclasista. Revista CEPAL, (129): 99137.

Brogna, Patricia (Comp.) (2008). Visiones y revisiones de la discapacidad. Fondo de Cultura Económica.

Cabra, Miguel, Francisco Bariffi \& Agustina Palacios (2007). Derechos humanos de las personas con discapacidad: La Convención Internacional de las Naciones Unidas. Editorial Universitaria Ramón Areces.

Castel, Robert (2006). La metamorfosis social de la cuestión social: Una crónica del salariado. Paidós.

Castel, Robert (2010). El ascenso de las incertidumbres. Trabajo, protecciones, estatuto del individuo. Fondo de Cultura Económica.

Castillo-Parada, Tatiana y Juan Carlos Cea-Madrid (2017). "Sin nosotros no hay derechos": apuntes sobre el Primer Encuentro Nacional por los Derechos Humanos de las personas en situación de discapacidad mental en Chile. Revista Latinoamericana en Discapacidad, Sociedad y Derechos Humanos, 1(1): 76 - 92.

Cea-Madrid, Juan Carlos (2021). "Cooperativismo loco": trabajo, comunidad e inclusión laboral en salud mental. Cuadernos de Relaciones Laborales, 39(1): 113 - 131. Doi: $10.5209 /$ crla.76228.

Cruz, Constanza, Magdalena Fadic, Edgardo Hernández \& Viviana Llambías (2015). Inclusión laboral: políticas y acción ¿Es lo que Chile necesita en el siglo XXI? Revista de Estudiantes de Terapia Ocupacional, 2(2): 81-94.

Chuaqui, Jorge (2015). Exclusión y discapacidad psíquica. En J. Chuaqui (Ed.). Sociología de la salud: Chile 2008-2015 (pp. 25-90). RIL Editores.

Ferrante, Carolina (2017). El éxito de la Teletón en Chile: paradoja y mensaje en la era de los derechos. Onteaiken. Boletín sobre Prácticas y Acción Colectiva, (23): 44-59.

Fundación Descúbreme (2019). Guía para la implementación de la ley de inclusión laboral. Herramientas para la inclusión laboral de personas con discapacidad en el sector privado. SENADIS - SOFOFA. 
Gomes, Raissa, Maria Carvalho-Freitas \& Marcos de Oliveira (2014). Relações entre significado do trabalho e percepção de suporte para pessoas com deficiência em organizações brasileiras. Estudos de Psicologia, 19(3): 217-226. Doi: 10.1590/ S1413-294X2014000300007.

Harvey, David (2007). Breve historia del neoliberalismo. Akal.

Humeres, Mónica (2019). “Gane usted y ayude a la Teletón”: mecanismos neoliberales en la gestión del bienestar. Convergencia, 26(81): 1-24. Doi: 10.29101/crcs. v26i81.11641.

Julián, Dasten (2014). Bases del modelo de valoración precario del trabajo en Chile: Acercamientos desde la política laboral y la cultura del trabajo. Sociológica, 29(81): 119-160.

Julián, Dasten \& Álvaro Galliorio (Comps.). (2018). Estudios del Trabajo desde el Sur. Ariadna Ediciones.

Montes, Catalina (2020). Políticas para la inclusión laboral de las personas con discapacidad en Chile. En C. Maldonado-Valera, M. L. Marinho \& C. Robles (eds.). Inclusión y cohesión social en el marco de la Agenda 2030 para el Desarrollo Sostenible: claves para un desarrollo social inclusivo en América Latina (pp.234239). Santiago: Comisión Económica para América Latina y el Caribe (CEPAL).

Muyor-Rodríguez, Jesús (2018). Nuevos significados de la discapacidad: De la igualdad de capacidades a la igualdad de derechos. Acciones e Investigaciones Sociales, 1(39): 33-55.

Narbona, Karina y Alexander Páez (2014). La acumulación flexible en Chile: aportes a una lectura socio-histórica de las transformaciones recientes del trabajo. Revista Pretérito Imperfecto, (2): 140-172.

Núnez-Parra, Lorena (2020). Discapacidad y trabajo: la individualización de la inclusión bajo lógicas coloniales contemporáneas. Nómadas, (52): 61-79.

Organización Internacional del Trabajo [OIT] (2015). Trabajo decente para personas con discapacidad: promoviendo derechos en la agenda global de desarrollo. Oficina Internacional del Trabajo.

Organización Internacional del Trabajo [OIT] (2013). Estudio: factores para la inclusión laboral de las personas con discapacidad. Organización Internacional del Trabajo.

Palacios, Agustina (2008). Modelo social de la discapacidad: orígenes, caracterización y plasmación en la Convención Internacional sobre los derechos de las personas con discapacidad. CERMI. 
Parker, Victoria, Josefina Blanch, Fernanda Ordenes \& Daniela Navarro (2020). Apoyos para la inclusión laboral en Santiago de Chile: Narrativa de expertos y experiencias de personas con discapacidad intelectual. Revista Latinoamericana en Discapacidad, Sociedad y Derechos Humanos, 4(2): 93-109.

Pereira-Silva, Nara Liana, Adelaine Vianna Furtado \& Jaqueline Andrade Ferreira Condé de Melo (2018). A Inclusão no Trabalho sob a Perspectiva das Pessoas com Deficiência Intelectual. Trends in Psychology, 26(2): 1003-1016.

Pino-Morán, Juan \& Pía Rodríguez-Garrido (2017). ¿Vivir para trabajar?: Activismo, Mujeres y Discapacidad en Chile. Intersticios. Revista sociológica de pensamiento crítico, 11(2): 185-198.

Pinto de Lima, Michelle, Nathália Vasconcelos Tavares, Mozar José Brito, \& Mônica Cappelle Carvalho Alves. (2013). O sentido do trabalho para pessoas com deficiência. RAM. Revista de Administração Mackenzie, 14(2): 42-68. Doi: 10.1590/ S1678-69712013000200003.

Ratto, Nicolás (2019). Estrategias sindicales y recursos de poder. Presentación y prueba empírica de un marco para el análisis de las variedades sindicales en Chile y en América Latina. Astrolabio, 23, 134-168.

Rojas, Oscar, Fernando Herrera, Natalie Muñoz \& Rocío Riffo (2019). Discapacidad intelectual e inserción laboral en Chile. Qualitas Investigaciones, 5(1): 37-42.

Ruiz-Bruzzone, Felipe (Ed.) (2016). Panorámica del trabajo en el Chile neoliberal. Editorial Universitaria.

Ruiz, Carlos \& Giorgio Boccardo (2014). Los chilenos bajo el neoliberalismo. Clase y conflicto social. Nodo XXI/El Desconcierto.

Servicio Nacional de la Discapacidad - SENADIS (2015). Segundo Estudio Nacional de la Discapacidad. SENADIS.

Soldatic, Karen \& Helen Meekosha (2012). Disability and Neoliberal State Formations. En Watson, N., A. Roulstone y C. Thomas (eds.). Routledge Handbook of Disability Studies (pp. 195-210). Nueva York: Routledge.

Sosa, Evelyn, Danitza Tenorio, Ximena Toro, Rodrigo Sepúlveda, Juan Aedo, Patricio Espinoza \& César Rodríguez (2015). Visión respecto a la legislación laboral chilena, desde sujetos en situación de discapacidad con participación política. Revista Chilena de Terapia Ocupacional, 15(1): 45-56.

Stecher, Antonio \& Vicente Sisto (2019). Trabajo y precarización laboral en el Chile neoliberal. Apuntes para comprender el estallido social de octubre 2019. En Araujo, K. (Coord.). Hilos tensados. Para leer el octubre chileno (pp. 37-82). Colección IDEA, Universidad de Santiago de Chile. 
Stecher, Antonio \& Lorena Godoy (2014). Transformaciones del trabajo, subjetividad e identidades, Lecturas psicosociales desde Chile y América Latina. RIL editores.

Vidal, Rubén \& Carolina Cornejo (2014). Aproximaciones al empleo con apoyo en Chile y Latinoamérica. Convergencia Educativa, (4): 99-111.

Vidal, Rubén, Carolina Cornejo \& Lucía Arroyo (2013). La inserción laboral de personas con discapacidad intelectual en Chile. Convergencia Educativa, (2): 93102.

Westermeier, Tanya, Mónica Salas, Sarai Jaramillo \& Ximena Torres (2018). Manual para la constitución de cooperativas inclusivas. Santiago: SENADIS - CIESCOOP - USACH.

\section{Sobre el autor}

Juan Carlos Cea Madrid es Psicólogo, Universidad de Santiago de Chile. Magíster en psicología, mención psicología comunitaria, Universidad de Chile. Investigador militante del Centro de Estudios Locos y activista del colectivo "Locos por nuestros derechos". Editor del libro "Por el derecho a la locura. La reinvención de la salud mental en América Latina”, Editorial Proyección (2018). Sus líneas de investigación son: salud mental y movimientos sociales en América Latina, trabajo y subjetividad en el Chile neoliberal. Correo Electrónico: jcarlos.ceamadrid@gmail.com. 


\title{
CUHSO
}

Fundada en 1984, la revista CUHSO es una de las publicaciones periódicas más antiguas en ciencias sociales y humanidades del sur de Chile. Con una periodicidad semestral, recibe todo el año trabajos inéditos de las distintas disciplinas de las ciencias sociales y las humanidades especializadas en el estudio y comprensión de la diversidad sociocultural, especialmente de las sociedades latinoamericanas y sus tensiones producto de la herencia colonial, la modernidad y la globalización. En este sentido, la revista valora tanto el rigor como la pluralidad teórica, epistemológica y metodológica de los trabajos.

\author{
EDITOR \\ Matthias Gloël \\ COORDINADORA EDITORIAL \\ Claudia Campos Letelier \\ CORRECTOR DE ESTILO Y DISEÑAdOR \\ Ediciones Silsag \\ TRADUCTOR, CORRECTOR LENGUA INGLESA \\ Aurora Sambolin Santiago \\ SITIO WEB \\ cuhso.uct.cl \\ E-MAIL \\ cuhso@uct.cl \\ LICENCIA DE ESTE ARTÍCULO \\ Creative Commons Atribución Compartir Igual 4.0 Internacional
}

